gras (21 et 22) et elle n'est que très imparfaitement éliminée lors du lavage du beurre (à l'opposé de la peroxydase), tandis que l'eau du beurre en présente une teneur relativement élevée. (Nous nous sommes convaincus de ces faits en préparant nous-mêmes du beurre avec de la crème de lait cru.) Ceci montre que la détermination de la phosphatase est le seul moyen chimique de se rendre compte si du beurre, ou un mélange de beurre et de margarine ou du "kwark »*, etc., est préparé avec du lait pasteurisé ou non.

KAY (13) détermine la phosphatase du beurre dans l'eau qui se sépare du beurre à $37^{\circ} \mathrm{C}$. Nous avons effectué cette détermination dans le beurre au moyen d'une méthode, que l'on peut aussi employer pour des mélanges de beurre et de margarine (qui parfois n'exsudent pas encore d'eau à $37^{\circ} \mathrm{C}$.) :

On chauffe $5 \mathrm{gr}$. de beurre non fondu avec $5 \mathrm{~cm}^{3}$ d'eau distillée et $0 \mathrm{~cm}^{2} 2$ d'huile sulforicinique dans un tube de culture fermé d'un bouchon de caoutchouc; chauffer à $37^{\circ} \mathrm{C}$. jusqu'à ce que le beurre soit à moitié fluide. On rend ensuite cette masse homogène en secouant énergiquement. On procède ensuite à une détermination $S$ et $W$ de l'émulsion de la façon décrite pour le lait.

Nous déterminons la phosphatase dans le "kwark" et les produits du même genre en broyant $5 \mathrm{gr}$. dans un mortier avec $10 \mathrm{~cm}^{3}$ d'eau et $0 \mathrm{cqm}^{3} 2$ d'huile sulforicinique et en traitant la suspension obtenue comme le lait.

(A suivre.)

\title{
ÉTUDE DE LA DILUTION DES CRÈMES ET DE SON INFLUENCE SUR LA COMPOSITION DES BABEURRES
}

\author{
par \\ JEAN PIEN
}

Ingénieur chimiste, Docteur ès Sciences, Directeur des Laboratoires de la Laiterie des Fermiers Réunis

La crème, comme le lait entier dont elle provient, peut être schématiquement représentée comme un mélange de lait écrémé et de matière grasse. Théoriquement, elle ne contient done pas d'eau - en dehors de l'eau de constitution du lait lui-même.

Or on constate pratiquement que beaucoup de crèmes sont plus ou moins diluées par un apport d'eau et l'étude technique du problème montre qu'il est difficile d'éviter complètement cette dilution. En effet : si le mouillage des crèmes ne résulte pour

(21) C. E. Rimpila, L. S. Patmer. Journ. Dairy Science, 18, 827, 1935.

(22) G. S. KАDT. Chem. Weekblad, 36, 427, 1939.

* Mélange de caséine et de matière grasse, obtenu par filtration de lait coagulé par acidification lactique ou par action de la présure. 
ainsi dire jamais d'une opération frauduleuse (qui serait sans profit pour son auteur puisque les transactions sur les crèmes s'effectuent sur leur teneur en matière grasse) en revanche, les opérations d'écrémage, de manutention, de transvasement des crèmes, etc., s'accompagnent nécessairement de rinȩages à l'eau dont le but, parfaitement louable, est d'assurer la récupération de toute la matière grasse. Ces eaux de rinçage, si elles sont purement et simplement mélangées à la erème - cas le plus fréquent dans les très petites exploitations - eausent une dilution évidente de la crème. Si elles sont écrémées, elles fournissent une "crème " récupérée dont la partie non grasse contient également de l'eau. Dans ce dernier cas, la masse totale de crème obtenue est, certes, moins " mouillée " que dans le cas précédent, mais elle l'est incontestablement.

Reconnaissons immédiatement que le problème ainsi soulevé serait supprimé si tous les rinçages étaient effectués à l'aide de lait écrémé, comme c'est le cas dans certains établissements importants. Mais cette manière de faire n'est pas généralisée et le problème se pose de l'étude de la dilution des crèmes et de ses conséquences.

$$
* * *
$$

Ce mouillage des crèmes - qu'il est difficile d'éviter complètement dans la pratique - comporte cependant des conséquences néfastes dont les principales sont les suivantes :

La maturation des crèmes, spontanée ou provoquée après pasteurisation, est modifiée ou compromise, surtout dans le cas de dilutions importantes.

Le babeurre (lait écrémé naturellement contenu dans la crème et évacué au cours du barattage) est évidemment dilué lui-même si la crème a été mouillée. Cette circonstance est désavantageuse si l'on se propose de fabriquer du babeurre en poudre (excès d'eau à évaporer) ou si le babeurre doit comporter un taux minimum d'extrait sec, en vue de la vente en nature. Notons que, dans le cas du babeurre, les eirconstances du traitement de la crème en beurrerie font apparaître une nouvelle cause possible de dilution qui vient s'ajouter à celles dont nous avons parlé à l'occasion de la production de la crème. Le problème se trouve done aggravé.

Pour ces deux raisons principales, il est désirable de tenter de produire et de manipuler les crèmes sans les additionner d'eau. Laissons de côté ce problème de technologie laitière et bornonsnous à en faire l'étude sur le plan analytique.

$$
\text { *** }
$$


Pour le chimiste chargé du contrôle des produits laitiers, il est indispensable :

De savoir reconnaître si une crème a été mouillée ;

De savoir déterminer le taux de ce mouillage d'après l'extrait isec de la crème ;

De savoir interpréter la composition des babeurres et, notamment, calculer le mouillage de la crème d'après l'extrait sec du babeurre.

Dans le but d'apporter une contribution à l'étude de ces divers points, nous nous proposons d'examiner les questions suivantes :

10 Etude de l'extrait sec théorique des crèmes pures;

$2^{\circ}$ Etude de l'influence du mouillage des crèmes sur leur extrait sec ;

$3^{\circ}$ Calcul du mouillage des crèmes d'après leur extrait sec;

$4^{\circ}$ Etude de l'influence du mouillage des crèmes sur l'extrait sec des babeurres;

$5^{\circ}$ Calcul du mouillage des crèmes d'après l'extrait sec des babeurres.

\section{$* * *$ \\ PREMIÈRE PARTIE \\ INCIDENCE DU MOUILLAGE DES CREMES \\ SUR LEUR EXTRAIT SEC}

\section{Extrait sec théorique des crèmes}

En principe, les analyses et les transactions sur la crème doivent avoir lieu en poids. Mais il peut arriver que l'on ait intérêt à connaître la composition volumétrique de la crème. C'est pourquoi nous distinguerons deux cas :

\section{$1^{\circ}$ Extrait sec théorique d'un kilogramme de crème.}

Cet extrait sec se compose :

a) $\mathrm{Du}$ poids de matière grasse pure contenue dans un kilogramme de crème ( $\mathrm{A}$ grammes) ;

b) Du poids de l'extrait sec de la partie non grasse de la crème qui n'est autre que du lait écrémé dont l'extrait sec vaut 90 à 95 grammes par litre (c'est-à-dire pour 1.034 à 1.036 grammes) ou encore 87 à 92 grammes par kilogramme (moyenne 90 grammes par kilogramme de lait écrémé ou 0 gr. 090 par gramme).

Le poids de la partie non grasse d'un kilogramme de crème est 
évidemment égal à $1.000-\mathrm{A}$, et l'extrait sec de cette partie non grasse, que nous appellerons $\mathrm{E}$, vaut :

$$
\mathrm{E}=0,090(1.000-\mathrm{A})
$$

ou :

$$
\mathrm{E}=90-0,09 \mathrm{~A} \text { gr. par kilogramme. }
$$

L'extrait sec total de la crème vaut donc :

$$
\mathrm{A}+0,09(1.000-\mathrm{A})
$$

ou :

$$
90+0,91 \text { A gr. par kilogramme. }
$$

\section{$2^{\circ}$ Extrait sec théorique d'un litrie de crème.}

Cet extrait sec se compose :

a) Du poids de matière grasse pure contenue dans un litre de crème (A grammes);

b) Du poids de l'extrait sec de la partie non grasse de la crème qui n'est autre que du lait écrémé dont l'extrait sec par litre peut: être considéré comme possédant une valeur moyenne de 93 grammes. Or, le volume de cette partie non grasse est égal à :

$$
1.000 \mathrm{~cm}^{3} \text { - volume de A. }
$$

Nous avons démontré antérieurement (1) qu'il fallait prendre 0,915 comme densité de la matière grasse pure dans le lait et la. crème, c'est-à-dire $\mathrm{A} / 0,915$ comme volume de la matière grasse.

Le volume de la partie non grasse d'un litre de crème vaut donc : $1.000-\mathrm{A} / 0,915$ et l'extrait sec de cette partie non grasse (E) doit être représenté par :

ou :

$$
\mathbf{E}=0,093(1.000-\mathrm{A} / 0,915)
$$

$$
\mathrm{E}=93-0,1016 \mathrm{~A} \text { gr. par litre }
$$

L'extrait sec total de la crème vaut done :

$$
\mathrm{A}+93-0,1016 \mathrm{~A}
$$

ou $\quad 93+0,8984$ A gr. par litre.

En résumé :

L'extrait sec dégraissé des crèmes - le seul qui nous intéresse ici - peut s'exprimer de la manière suivante en fonction de la teneur de la crème en matière grasse.

Extrait dégraissé par kilogramme de crème :

$$
\left.\mathrm{E}=0,090(1.000-\mathrm{A}) \quad \text { (formule } \mathrm{n}^{\circ} \mathrm{i}\right)
$$

(1) Le Lait, 1938, p. 582 . 
Extrait dégraissé par litre de crème :

$$
\mathrm{E}=93-0,1016 \mathrm{~A} \quad \text { (formule } \mathrm{n}^{\circ} 2 \text { ) }
$$

Le terme A représente la teneur de la crème en matière grasse par kilogramme dans la formule $n^{0} 1$ et en matière grasse par litre dans la formule $n^{0} 2$.

\section{$3^{\circ}$ Exemples d'application des formules précédentes.}

\begin{tabular}{c|c|c|c}
\hline \hline \multicolumn{2}{c|}{ Résultats par kilogramme de erème } & \multicolumn{2}{|c}{ Résultats par litre de crème } \\
\cline { 1 - 2 } $\begin{array}{c}\text { Richesse } \\
\text { en matière grasse } \\
\text { par kilogramme (A) }\end{array}$ & $\begin{array}{c}\text { Extrait dégraissé } \\
\text { par kilogramme }\end{array}$ & $\begin{array}{c}\text { Richesse } \\
\text { en matière grasse } \\
\text { par litre (A) }\end{array}$ & $\begin{array}{c}\text { Extrait dégraissé } \\
\text { par litre (E) }\end{array}$ \\
200 & 72,0 & 200 & 72,7 \\
250 & 67,5 & 250 & 67,6 \\
300 & 63,0 & 300 & 62,5 \\
350 & 58,5 & 350 & 57,4 \\
400 & 54,0 & 400 & 52,3 \\
450 & 49,5 & 450 & 47,3 \\
500 & 45,0 & 500 & 42,2 \\
\hline
\end{tabular}

Nota. - Ces chiffres théoriques comportent une légère part d'incertitude du fait que nous avons admis pour l'extrait sec moyen du lait écrémé 93 grammes par litre avec une densité moyenne de 1,035 .

Or, il est évident que certains laits entiers fournissent des laits écrémés - donc une partie non grasse de la crème - ayant des caractéristiques quelque peu inférieures. Ainsi, en prenant 90 grammes d'extrait ou 1,034 de densité pour le lait écrémé, les chiffres du tableau précédent se trouveraient diminués de 1 gr. 5 à 2 grammes par litre ou par kilogramme.

Done, quand on comparera les résultats de l'analyse aux chiffres théoriques obtenus par l'emploi des formules précédentes, on ne conclura avec certitude au mouillage, que si les chiffres constatés sont inférieurs de quelques grammes ou davantage aux chiffres calculés. Cette légère incertitude - la même d'ailleurs que l'on retrouve dans l'interprétation du mouillage des laits et pour les mêmes raisons - ne nuit en rien au dépistage des mouillages égaux ou supérieurs à $5 \%$.

II. Détermination de l'extrait sec des crèmes par l'analyse 10 Méthode approchée.

a) Matière grasse : on dosera la matière grasse par litre ou 
par kilogramme en opérant par la méthode "Gerber par dilution » en partant de $10 \mathrm{~cm}^{3}$ ou de 10 grammes de crème ;

b) Extrait sec total : par évaporation au bain-marie et séchage à poids constant de $10 \mathrm{~cm}^{3}$ ou 10 grammes de crème comme dans. le cas des laits ;

c) Extrait sec dégraissé: par différence.

\section{$2^{\circ}$ Méthode exacte (uniquement par kilogramme de crème).}

a) Matière grasse : on dosera la matière grasse par kilogramme de crème en utilisant la méthode "Gottlieb par centrifugation » avec trois extractions consécutives appliquée à un gramme de crème ;

b) Extrait sec total : par évaporation au bain-marie et séchage à poids constant de 10 grammes de crème;

c) Extrait sec dégraissé : par différence.

Nota. - Pour l'application de ces méthodes analytiques, on pourra se reporter à l'étude que nous avons publiée de ces questions (1).

\section{Influence du mouillage sur l'extrait sec des crèmes}

Avant de montrer comment on peut calculer le taux de mouillage des crèmes connaissant leur extrait sec dégraissé, il est intéressant de se demander comment varie celui-ci en fonction du mouillage.

Pour la commodité de l'exposé et pour se rapprocher, dans. l'expression des résultats, des conditions de la pratique, nous appellerons "mouillage» (représenté par M) le volume d'eau en centimètres cubes ajouté à un litre ou un kilogramme de crème.

Soit une crème pure, de richesse en matière grasse représentée par A grammes (par kilogramme ou par litre) et d'extrait secdégraissé $\mathrm{E}$ (par kilogramme ou par litre). Cette valeur $\mathrm{E}$ est facile à connaître dans chaque eas grâce aux formules $n^{0} 1$ et $n^{\circ} 2$.

Appelons $E^{\prime}$ l'extrait sec dégraissé de la crème diluée (par kilogramme ou par litre). Il s'agit done de calculer $\mathbf{E}^{\prime}$ en fonction. de $\mathbf{M}$ et de $\mathbf{A}$ (ou de $\mathbf{E}$ ).

Ces divers éléments ( $\left.\mathrm{A}, \mathrm{E}, \mathrm{E}^{\prime}, \mathrm{M}\right)$ doivent être considérés,

(1) Le Lait, janvier-mars 1943, p. 18. 
ensemble, comme se rapportant tous au kilogramme de crème, ou. bien tous au litre de crème.

La dilution a consisté à ajouter $\mathbf{M}$ grammes d'eau à 1.000 grammes de crème (par exemple).

La quantité d'eau contenue dans 1.000 grammes de crème diluée vaut donc :

$$
\frac{1.000 \times \mathrm{M}}{1.000+\mathrm{M}}
$$

La quantité de matière grasse contenue dans 1.000 grammes. de crème diluée vaut :

$$
\frac{1.000 \times \mathrm{A}}{1.000+\mathrm{M}}
$$

On conçoit évidemment que l'extrait sec dégraissé $\mathbf{E}^{\prime}$ de la crème diluée aura pour valeur :

$$
\frac{1.000 \times \mathrm{E}}{1.000+\mathrm{M}}
$$

Il est cependant intéressant de le démontrer directement.

Cette crème diluée peut être considérée comme contenant, par kilogramme, à côté de cette eau et de cette matière grasse, un certain poids de lait écrémé pur égal à :

$$
1.000-\frac{1.000 \times \mathrm{A}}{1.000+\mathrm{M}}-\frac{1.000 \times \mathrm{M}}{1.000+\mathrm{M}} \text { grammes par kilogramme }
$$

ou :

$$
1.000 \times\left(\frac{1.000-\mathrm{A}}{1.000+\mathrm{M}}\right)
$$

Or, un kilogramme de lait écrémé possède un extrait sec moyen de 90 grammes.

L'extrait sec du lait écrémé de la crème - c'est-à-dire l'extrait sec dégraissé $\mathrm{E}^{\prime}$ de la crème elle-même - vaut donc par kilogramme :

$$
\mathbf{E}^{\prime}=\frac{1.000 \times 0,090(1.000-\mathrm{A})}{1.000+\mathrm{M}}
$$

Mais 0,090 (1.000 - A) n'est autre que $\mathrm{E}$ (voir formule no 1). Done :

$$
E^{\prime}=\frac{1.000 \times E}{1.000+M} \quad\left(\text { formule } n^{\circ} 3\right)
$$

La formule est évidemment la même, que les calculs soient effectués par kilogramme ou par litre de crème - ce que le calcul direct montre d'ailleurs aisément - à condition que tous les élé- 
ments soient exprimés ensemble de la même manière (par litre ou par kilogramme).

Notons enfin que l'on pourrait, de cette formule $n^{0} 3$, tirer la valeur du mouillage en fonction de l'extrait constaté sur la crème diluée ( $\left.\mathrm{E}^{\prime}\right)$, et de l'extrait sec initial de la crème non diluée (E) tiré lui-même de la connaissance de $A$, taux de matière grasse de la crème initiale non diluée.

On aboutirait à :

$$
\mathbf{M}=\frac{1.000\left(\mathbf{E}-\mathbf{E}^{\prime}\right)}{\mathbf{E}^{\prime}}
$$

Mais cette formule serait sans intérêt pratique attendu que lorsqu'on examine une crème diluée où l'on dose $\mathbf{E}^{\prime}$, on ignore la composition de la crème initiale $(\mathrm{A}, \mathrm{E})$.

Le calcul du mouillage n'est vraiment possible et utile que si l'on part des éléments de la crème diluée, les seuls que nous puissions connaître par l'analyse.

Montrons néanmoins, sur des exemples pratiques, ce que devient l'extrait sec initial E d'une crème pure, de richesse $A$, sous l'influence de mouillages variés $\mathbf{M}$ définis comme il a été dit :

\begin{tabular}{|c|c|c|c|c|c|c|}
\hline \multirow{2}{*}{$\begin{array}{c}\text { Richesse } \\
\text { initiale en } \\
\text { matière } \\
\text { grasse par } \\
\text { kilogram. } \\
\text { me (A) }\end{array}$} & \multirow{2}{*}{$\begin{array}{l}\text { Extrait } \\
\text { dégraissé } \\
\text { initial par } \\
\text { kilogram- } \\
\text { me (E) }\end{array}$} & \multicolumn{5}{|c|}{$\begin{array}{l}\text { Extrait dégraissé (E') d'un kilogramme de crème diluée } \\
\text { pour des mouillages par kilogramme de erème initiale de }\end{array}$} \\
\hline & & $100 \mathrm{~cm}^{3}$ & $200 \mathrm{~cm}^{3}$ & $300 \mathrm{~cm}^{3}$ & $400 \mathrm{~cm}^{3}$ & $500 \mathrm{~cm}^{3}$ \\
\hline 200 & 72,0 & 65,4 & 60,0 & 55,4 & 51,4 & 48,0 \\
\hline 250 & 67,5 & 61,4 & 56,2 & 51,9 & 48,2 & 45,0 \\
\hline 300 & 63,0 & 57,3 & 52,5 & 48,4 & 45,0 & 42,0 \\
\hline 350 & 58,5 & 53,2 & 48,7 & 45,0 & 41,8 & 39,0 \\
\hline 400. & 54,0 & 49,1 & 45,0 & 41,5 & 38,5 & 36,0 \\
\hline 450 & 49,5 & 45,0 & 41,2 & 38,1 & 35,3 & 33,0 \\
\hline 500 & 45,0 & 40,9 & 37,5 & 34,6 & 32,1 & 30,0 \\
\hline
\end{tabular}

\section{Calcul du mouillage des crèmes (d'après l'extrait sec des crèmes diluées)}

Le véritable problème pratique est le suivant : connaissant les caractéristiques de la crème diluée, en déduire le mouillage de la erème initiale, c'est-à-dire, ici, et par convention, la quantité d'eau ajoutée à un litre ou un kilogramme de crème initiale. 
Appelons :

$A^{\prime}$ la richesse en matière grasse par kilogramme de la crème diluée ;

$\mathbf{E}^{\prime}$ l'extrait sec par kilogramme de la crème diluée ;

M la quantité d'eau ajoutée par kilogramme ou par litre de crème initiale:

\section{$1^{0}$ Calcul du mouillage par kilogramme de crème.}

Comme précédemment, la dilution a consisté à ajouter $\mathrm{M}$ grammes d'eau à 1.000 grammes de crème initiale.

Le poids total d'eau contenu dans 1.000 grammes de crème diluée est égal à $\frac{1.000 \times M}{1.000+M}$.

Le poids de matière grasse contenu dans 1.000 grammes de crème diluée, vaut $\mathrm{A}^{\prime}$.

La crème diluée peut donc être considérée comme contenant par kilogramme, à côté de $A^{\prime}$ grammes de matière grasse, la quantité d'eau précédente et un certain poids de lait écrémé pur égal à :

$$
1.000-\mathrm{A}^{\prime}-\frac{1.000 \times \mathrm{M}}{1.000+\mathrm{M}} \text { grammes }
$$

L'extrait see du lait écrémé étant, en moyenne, de 90 grammes par kilogramme, l'extrait sec du lait écrémé de la crème, c'est-à-dire l'extrait dégraissé $\mathbf{E}^{\prime}$ de la crème elle-même, vaudra. done par kilogramme :

$$
\begin{aligned}
& \mathbf{E}^{\prime}=0,090\left(1.000-A^{\prime}-\frac{1.000 \times M}{1.000+M}\right) \\
& \text { ou }=0,090\left(1.000-A^{\prime}\right)-\frac{90 \times M}{1.000+M}
\end{aligned}
$$

d'où l'on tire, tous calculs faits :

$$
\mathrm{M}=1.000\left(\frac{90}{0,09 \mathrm{~A}^{\prime}+\mathrm{E}^{\prime}}-1\right) \quad \text { (formule } \mathrm{n}^{0} 4 \text { ) }
$$

Vérification de la formule sur un exemple numérique :

Soit une crème pure à 400 grammes de matière grasse par kilogramme. Ajoutons-lụi $100 \mathrm{~cm}^{3}$ d'eau par kilogramme de crème. La matière grasse de la crème diluée $\left(\mathrm{A}^{\prime}\right)$ est égale à :

$$
\frac{1.000 \times 400}{1.100}=363 \text { gr. } 6
$$

L'extrait sec initial de la crème pure était, d'après la formule 
$n^{\circ} 1$, égal à 54 grammes par kilogrammes. Du fait de la dilution, il est devenu :

$$
\mathrm{E}^{\prime}=\frac{54 \times 1.000}{1.100}=49 \mathrm{gr} .09
$$

Portons ces valeurs de $\mathrm{A}^{\prime}$ et de $\mathrm{E}^{\prime}$ dans la formule $\mathrm{n}^{0} 4$. Nous trouvons : $\mathrm{M}=100,05$ (pour $100 \mathrm{~cm}^{3}$ réellement ajoutés).

La formule no 4 est donc bien exacte.

\section{$2^{\circ}$ Calcul du mouillage par litre de crème.}

La formule $n^{\circ} 4$ nous permet de calculer la quantité d'eau qui a été introduite dans un kilogramme de crème pure initiale. On peut donc en déduire la richesse initiale $A$ en matière grasse par kilogramme de crème pure :

$$
\mathrm{A}=\mathrm{A}^{\prime} \times \frac{1.000+\mathrm{M}}{1.000}
$$

Connaissant $\mathbf{A}$, on peut en déduire la densité de la crème initiale à l'aide de la formule :

$$
\mathrm{D}=\frac{1.035}{1.000+0,1311 \mathrm{~A}}
$$

Enfin, connaissant cette densité et M. par kilogramme, on peut en déduire $\mathrm{M}^{\prime}$ par litre, e'est-à-dire le volume d'eau ajouté à un litre de crème :

$$
\begin{gathered}
M^{\prime}=M \times D\left(\text { formule } n^{\circ} 4 b i s\right) \\
*^{*}
\end{gathered}
$$

\section{DEUXIEME PARTIE}

\section{INCIDENCE DU MOUILLAGE DES CRÈ MES SUR L'EXTRAIT SEC} DES BABEURRES

\section{Extrait sec théorique des babeurres}

Le babeurre n'étant pas autre chose que le lait écrémé contenu dans la crème, son extrait sec théorique (cas d'une crème non diluée) est égal à celui du lait écrémé lui-même, c'est-à-dire compris entre 90 et 95 grammes par litre suivant les saisons et les régions d'origine du lait initial.

Dans tous nos calculs, nous prenons le chiffre moyen de 93 grammes par litre.

(1) Voir notre étude antérieure, Le Lait, 1938, p. 582. 


\section{Influence du mouillage des crèmes sur l'extrait sec des babeurres}

Le movillage de la crème se traduit évidemment par une dilution correspondante du babeurre. Mais le pourcentage d'eau retrouvée dans le babeurre (où l'eau de la crème passe intégralement) sera relativement plus élevé que le pourcentage d'eau ajouté à la crème, et cela suivant la richesse en matière grasse de la crème.

Il est done intéressant de se demander comment varie l'extrait sec du babeurre en fonction du mouillage d'une crème définie par ses caractéristiques initiales ou, mieux, par ses caractéristiques finales, c'est-à-dire après dilution.

\section{Calcul de l'extrait sec du babeurre en fonction des carac- téristiques initiales de la crème pure.}

La partie non grasse d'un litre de crème pure (c'est-à-dire le futur babeurre) possède un volume égal à :

où

$$
1.000-\mathrm{A} / 0,915 \mathrm{~cm}^{3}
$$

$\mathrm{A}=$ matière grasse par litre de crème pure.

Le dosage de la matière grasse de la crème ne pouvant être obtenu avec précision que par une analyse "au kilogramme», on ramènera la richesse trouvée par kilogramme $\left(A_{k}\right)$ à la richesse au litre (A) en utilisant la formule suivante :

$$
A=\frac{1.035 \mathrm{~A}^{\mathrm{k}}}{1.000+0,1311 \mathrm{~A}_{\mathrm{k}}}
$$

L'extrait sec de ce babeurre, assimilable à du lait écrémé, est donc égal à $\mathbf{E}$, extrait sec dégraissé de la crème, fourni par l'analyse ou calculé à partir de A à l'aide de la formule $n^{0} 2$.

Si l'on ajoute $M$ centimètres cubes d'eau à ce litre de crème, l'extrait sec du babeurre total n'est pas modifié, mais le volume de ce babeurre est augmenté de M. et devient :

$$
1.000-\mathrm{A} / 0,915+\mathrm{M}
$$

L'extrait see du babeurre, par litre de babeurre (e) śera done égal à :

$$
\mathrm{e}=\frac{\mathrm{E}}{1.000-\frac{\mathrm{A}}{0,915}+\mathrm{M}} \times 1.000
$$

(1) Le Lait, 1938, p. 582. 
Mais 1.000 - A/0,915 n'est pas autre chose que E/0,093 d'après la formule $n^{0} 2$.

Nous pouvons done écrire:

$$
\left.\mathrm{e}=\frac{1.000 \mathrm{E}}{\mathrm{E} / 0,093+\mathrm{M}}=\frac{93 \mathrm{E}}{\mathrm{E}+0,093 \mathrm{M}} \quad \text { (formule } \mathrm{n}^{\circ} 5\right)
$$

Telle est done la valeur de l'extrait sec du babeurre par litre de babeurre en fonction de l'extrait sec dégraissé $\mathrm{E}$ d'un litre de crème pure initiale - ou bien, en fonction de $\mathrm{A}$ d'où l'on passe à $\mathrm{E}$ à l'aide de lá formule $n^{\circ} 2$ - et du taux de dilution $M$ que l'on se propose d'appliquer à cette crème (volume d'eau ajouté à un litre de crème).

De même que la formule- $n^{\circ} 3$, cette formule $n^{\circ} 5$ ne présente qu'un intérêt théorique, attendu que lorsqu'on examine une crème diluée dont on veut ainsi prévoir la richesse en extrait sec du babeurre, on ignore la composition de la crème initiale. Le calcul, à priori, de l'extrait sec du babeurre, ou bien le calcul du mouillage de la crème initiale en partant de l'extrait see réel de ce babeurre, ne présente d'intérêt pratique que si l'on part des éléments de la crème diluée, les seuls que nous puissions connaître par l'analyse.

Montrons néanmoins, sur des exemples pratiques, ce que devient l'extrait see du babeurre d'une crème pure de richesse initiale $A$, sous l'influence des mouillages variés $\mathbf{M}$ définis comme il a été dit :

\begin{tabular}{|c|c|c|c|c|c|c|}
\hline \multirow{2}{*}{$\begin{array}{c}\text { Richesse } \\
\text { initiale en } \\
\text { matière } \\
\text { grasse par } \\
\text { litre (A). }\end{array}$} & \multirow{2}{*}{$\begin{array}{c}\text { Extrait } \\
\text { dégraissé } \\
\text { initial de la } \\
\text { erème (E) } \\
\text { par litre }\end{array}$} & \multicolumn{5}{|c|}{$\begin{array}{c}\text { Extrait sec des babeurres (par litre) pour des mouillages } \\
\text { par litre de crème de : }\end{array}$} \\
\hline & & $100 \mathrm{~cm}^{3}$ & $200 \mathrm{~cm}^{3}$ & $300 \mathrm{~cm}^{3}$ & $400 \mathrm{~cm}^{3}$ & $500 \mathrm{~cm}^{3}$ \\
\hline 200 & 72,7 & 82,4 & 74,0 & 67,2 & 61,5 & 56,7 \\
\hline 250 & 67,6 & 81,7 & 72,9 & 65,8 & 60,0 & 55,1 \\
\hline 300 & 62,5 & 80,9 - & 71,6 & 64,3 & 58,3 & 53,3 \\
\hline 350 & 57,4 & 80,0 & 70,2 & 62,5 & 56,4 & 51,4 \\
\hline 400 & 52,3 & 78,9 & 68,6 & 60,6 & 54,3 & 49,2 \\
\hline 450 & 47,3 & 77,7 & 66,7 & 58,4 & 52,0 & 46,8 \\
\hline 500 & 42,2 & 76,2 & 64,5 & 55,9 & 49,4 & 44,2 \\
\hline
\end{tabular}

\section{$2^{\circ}$ Calcul de l'extrait sec du babeurre en fonction des carac- téristiques de la crème diluée.}

Ce problème, d'un intérêt pratique immédiat, consiste à calculer à l'avance l'extrait sec du babeurre qui sera fourni par une 
crème diluée dont on connaît le taux de matière grasse par kilogramme et l'extrait dégraissé par kilogramme, ignorant tout de la crème pure initiale dont elle provient.

Nous devons nécessairement partir de données obtenues par l'analyse au kilogramme de crème - les résultats obtenus par litre étant toujours, on le sait, plus ou moins entachés d'erreur. En revanche, l'extrait sec du babeurre doit être exprimé soit par kilogramme de babeurre, soit plus généralement, par litre de babeurre. Il en résultera, dans ce dernier cas, une difficulté supplémentaire que nous étudierons.

Partant de données "pondérales ", exprimons l'extrait sec du babeurre des deux manières :

a) Extrait sec du babeurre exprimé par kilogramme de babeurre.

Nous partons done d'une crème diluée dont nous connaissons, par l'analyse, la matière grasse par kilogramme $\left(A^{\prime}\right)$ et l'extrait dégraissé par kilogramme ( $\left.\mathbf{E}^{\prime}\right)$. Il s'agit de calculer l'extrait sec du babeurre (e) par kilogramme de babeurre en fonction de $A^{\prime}$ et de $\mathbf{E}^{\prime}$.

L'extrait sec dégraissé $\mathbf{E}^{\prime}$ n'est autre que l'extrait sec du futur babeurre contenu dans un kilogramme de crème. Or, le poids du babeurre dilué contenu dans ce kilogramme de crème diluée, est évidemment égal à $1.000-A^{\prime}$. L'extrait sec (e) du babeurre par kilogramme de babeurre dilué, sera done égal à :

$$
\mathrm{e}=\frac{\mathrm{E}^{\prime}}{1.000-\mathrm{A}^{\prime}} \times 1.000 \quad \text { (formule } \mathrm{n}^{\circ} 6 \text { ) }
$$

b) Extrait sec du babeurré exprimé par litre de babeurre.

En général, il est plus intéressant de connaître l'extrait sec du babeurre par litre que par kilogramme (les quantités de babeurre sont, en effet, exprimées plutôt en volume qu'en poids). Aussi, est-il nécessaire de savoir calculer cet extrait sec par litre en partant; néanmoins, des earactères par kilogŕamme de la crème diluée, comme dans le cas précédent, puisque c'est là le seul moyen de les obtenir avec certitude et précision.

L'extrait sec dú babeurre contenu dans un kilogramme de crème diluée, n'est autre que l'extrait dégraissé de cette crème ellemême $\left(\mathrm{E}^{\prime}\right)$. Or, on sait qu'un kilogramme de babeurre pur possède un extrait sec de 90 grammes environ, e'est-à-dire - la densité du babeurre étant égale, en moyenne, à 1,035 - de $90 \times 1,035$ par litre ou $0,09 \times 1,035$ par centimètre cube.

Le volume de babeurre pur contenu dans un kilogramme de crème diluée, est done égal à : $\mathrm{E}^{\prime} / 0,09 \times 1,035$. 
Le volume du babeurre dilué contenu dans un kilogramme de crème, est égal au volume précédent majoré du volume d'eau contenu dans un kilogramme de crème. Ce volume $\mathbf{M}^{t}$ vaut : $1.000 \times \mathrm{M}$ $\overline{1.000+M^{\circ}}$.

En remplaçant $M$ par sa valeur issue de la formule $n^{0} 4$, nous trouvons, tous calculs faits, $\mathbf{M}^{\prime}=1.000-\mathrm{A}^{\prime}-\mathbf{E}^{\prime} / 0,09$.

Le volume total du babeurre dilué d'un kilogramme de crème, est done égal à :

$$
\begin{gathered}
\mathrm{V}=\frac{\mathbf{E}^{\prime}}{0,09 \times 1,035}+1.000-\mathrm{A}^{\prime}-\frac{\mathrm{E}^{\prime}}{0,09} \\
=1.000-\mathrm{A}^{\prime}-\frac{35}{93} \mathbf{E}^{\prime}
\end{gathered}
$$

L'extrait sec dégraissé $\mathrm{E}^{\prime}$ de la crème, c'est-à-dìre l'extrait du babeurre de la crème, est réparti dans ce volume total. En le ramenant à un litre de babeurre, nous aurons :

$$
\mathrm{e}=\frac{\mathrm{E}^{\prime}}{\mathrm{V}} \times 1.000=\frac{\mathrm{E}^{\prime} \times 1.000}{1.000-\mathrm{A}^{\prime}-\frac{35}{93}} \mathbf{E}^{\prime}
$$

soit, tous calculs faits :

$$
\mathrm{e}=\frac{93.000 \mathrm{E}^{\prime}}{93\left(1.000-\mathrm{A}^{\prime}\right)-35 \mathrm{E}^{\prime}} \quad\left(\text { formule } \mathrm{n}^{0} 7\right)
$$

Telle est la valeur, en grammes par litre, de l'extrait dégraissé du babeurre dilué provenant d'une crème diluée dont on connaît la matière grasse par kilogramme $\left(\mathrm{A}^{\prime}\right)$ et l'extrait sec dégraissé $\left(\mathrm{E}^{\prime}\right)$.

\section{$3^{\circ}$ Mouillage centésimal du babeurre.}

Si l'on admet que l'extrait sec théorique du babeurre pur, comme celui du lait écrémé, est égal, en moyenne, à 93 grammes par litre, de la valeur (e) trouvée pour l'extrait du babeurre à l'aide des formules précédentes, on peut calculer le taux de mouillage $\%$ du babeurre lui-même. Il vaut :

$$
\text { mouillage } \left.\%=\frac{(93-\mathrm{e}) \times 100}{93} \quad \text { (formule } \mathrm{n}^{\circ} 8\right)
$$

\section{Calcul du mouillage des crèmes d'après l'extrait sec des babeurres}

L'un des problèmes pratiques les plus intéressants à résoudre dans le domaine qui nous occupe ici est le suivant : le dosage direct de l'extrait sec dégraissé d'un babeurre (e) nous montre qu'il est 
mouillé (inférieur à 93 grammes par litre). Ce babeurre provient donc d'une crème diluée. Connaissant la richesse $\mathrm{A}^{\prime}$ en matière grasse par kilogramme de la crème diluée, en déduire le mouillage de la crème initiale.

Reprenons les calculs qui nous ont menés à la formule $\mathrm{n}^{0} 7$ et exprimons la valeur de (e) en fonction de $\mathrm{M}$.

L'extrait sec du babeurre contenu dans un kilogramme de crème diluée est égal à l'extrait sec dégraissé de la crème elle-même, c'est-à-dire $\mathbf{E}^{\prime}$.

Le volume de ce babeurre supposé pur est égal, avons-nous dit; à :

$$
\frac{\mathbf{E}^{\prime}}{0,09 \times 1,035} \text { ou } \frac{\mathbf{E}^{\prime}}{0,093}
$$

La quantité d'eau contenue dans un kilogramme de crème diluée est égale à :

$$
\frac{1.000 \times \mathrm{M}}{1.000+\mathrm{M}}
$$

Donc, le volume total du babeurre dilué est égal à :

$$
\mathrm{V}=\frac{\mathrm{E}^{\prime}}{0,093}+\frac{1.000 \times \mathrm{M}}{1.000+\mathrm{M}} \text { en centimètres cubes }
$$

ou

$$
V=\frac{\mathbf{E}^{\prime}(1.000+\mathrm{M})+93 \mathrm{M}}{0,093(1.000+\mathrm{M})}
$$

L'extrait sec de ce volume étant $\mathbf{E}^{\prime}$, l'extrait (e) du babeurre par litre de babeurre est égal à :

$\mathrm{e}=\frac{\mathrm{E}^{\prime}}{\mathrm{V}} \times 1.000=1.000 \times \frac{\mathrm{E}^{\prime} \times 0,093(1.000+\mathrm{M})}{\mathrm{E}^{\prime}(1.000+\mathrm{M})+93 \mathrm{M}}=\frac{93 \mathrm{E}^{\prime}(1.000+\mathbf{M})}{93 \mathrm{M}+\mathrm{E}^{\prime}(1.000+\mathrm{M})}$

d'où l'on tire :

$$
\left.\mathrm{M}=\frac{1.000 \mathrm{E}^{\prime}(93-\mathrm{e})}{93 \mathrm{e}-\mathrm{E}^{\prime}(93-\mathrm{e})} \quad \text { (formule } \mathrm{n}^{\circ} 9\right)
$$

Cette formule permet de calculer M (quantité d'eau ajoutée à un kilogramme de crème initiale) en fonction de $\mathbf{E}^{\prime}$ (extrait dégraissé - de la crème diluée par kilogramme) et de e (extrait dégraissé du babeurre par litre). Or, il est beaucoup plus intéressant d'exprimer ce résultat en fonction de ' $A^{\prime}$ (matière grasse par kilogramme de la crème diluée) plutôt qu'en fonction de $\mathbf{E}^{\prime}$, car le dosage de la matière grasse est une opération plus rapide et plus aisée que le dosage de l'extrait sec dégraissé. 
Pour exprimer $M$ en fonction de $A^{\prime}$ et de e, il faut remonter à l'expression précédente donnant e en fonction de $\mathbf{M}$ et de $\mathbf{E}^{\prime}$.

Nous avions :

$$
\mathrm{e}=\frac{93 \mathrm{E}^{\prime}(\mathbf{1} .000+\mathrm{M})}{93 \mathrm{M}+\mathrm{E}^{\prime}(1.000+\mathrm{M})}
$$

Il s'agit done d'exprimer E' en fonction de $\mathrm{A}^{\prime}$ et de porter cette nouvelle valeur dans l'équation ci-dessus. Or, la formule $n^{\circ} 4$ nous permet de calculer $\mathrm{E}^{\prime}$ en fonction de $\mathrm{A}^{\prime}$. Tous calculs faits, nous obtenons :

$$
\mathrm{E}^{\prime}=\frac{90.000-0,09 \mathrm{~A}^{\prime}(1.000+\mathrm{M})}{1.000+\mathrm{M}}
$$

Portons cette valeur dans l'équation précédente de $e$. Il vient :

$$
\mathrm{e}=\frac{93\left[90.000-0,09 \mathrm{~A}^{\prime}(1.000+\mathrm{M})\right]}{93 \mathrm{M}+90.000-0,09 \mathrm{~A}^{\prime}(1.000+\mathrm{M})}
$$

d'où nous tirons finalement :

$$
\left.\mathbf{M}=\frac{90\left(1.000-\mathrm{A}^{\prime}\right)(93-\mathrm{e})}{93 \mathrm{e}+0,09 \mathrm{~A}^{\prime}(93-\mathrm{e})} \quad \text { (formule } \mathrm{n}^{\circ} 10\right)
$$

Cette formule permet de calculer, comme la formule $\mathrm{n}^{\circ} 4$, la quantité d'eau introduite dans un kilogramme de crème pure initiale, mais en partant d'autres données qui sont:

L'extrait sec dégraissé du babeurre par litre de babeurre (e).

La matière grasse par kilogramme de la crème diluée $\left(A^{\prime}\right)$.

On pourrait passer du mouillage par kilogramme de crème ainsi obtenu au mouillage par litre de crème $\left(\mathbf{M}^{\prime}\right)$ en opérant comme on l'a fait à l'oceasion de l'établissement de la formule $\mathrm{n}^{0} 4$ bis $\left(\mathbf{M}^{\prime}=\mathbf{M} \times \mathrm{D}\right)$.

Application particulière : taux de movillage maximum d'une crème pour obtenir un babeurre possédant un extrait sec minimum fixé à l'avance.

Supposons que l'on veuille obtenir, dans tous les cas; un babeurre ne titrant pas moins de 65 grammes d'extrait sec dégraissé par litre. En introduisant cette valeur $(\mathrm{e}=65)$ dans la formule no 5 , nous en déduirons la valeur de $\mathbf{M}$, c'est-à-dire la quantité d'eau qu'il ne faudra pas dépasser par litre de crème initiale.

Nous aurons :

$$
\mathrm{M}=\frac{\mathbf{E}(93-\mathrm{e})}{0,093 \mathrm{e}}
$$


ou encore, puisque $\mathrm{E}=0,093(1.000-\mathrm{A} / 0,915)$ :

$$
\mathrm{M}=\frac{(1.000-\mathrm{A} / 0,915)(93-\mathrm{e})}{\mathrm{e}} \quad \text { (formule } \mathrm{n}^{\circ} 5 \text { bis) }
$$

ou, ici, pour e $=65$

$$
\mathrm{M}=0,43\left(1.000-\frac{\mathrm{A}}{0,915}\right)
$$

Cette quantité d'eau varie naturellement suivant la richesse A en matière grasse de la crème initiale ou, ce qui revient au même, suivant son extrait sec dégraissé $\mathbf{E}$. suivants :

L'application de cette formule conduit aux résultats

Pour une crème à 200 gr. de M. G. par L. $(E=72,7), M=336 \mathrm{~cm}^{3} 6$ d'eau. Pour une crème à $300 \mathrm{gr}$. de $M$. G. par L. $(E=62,5), M=289 \mathrm{~cm}^{3} 3$ d'eau. Pour une crème à $400 \mathrm{gr}$. de $\mathrm{M}$. G. par L. $(\mathrm{E}=52,3), \mathrm{M}=242 \mathrm{~cm}^{3} 1$ d'eau. Pour une crème à 500 gr. de $M$. G. par L. $(E=42,2), M=195 \mathrm{~cm}^{3} 4$ d'eau.

Pour des crèmes d'une richesse moyenne de 400 à 500 grammes de matière grasse par litre, cela signifie que pour avoir du babeurre à 65 grammes d'extrait see dégraissé, il ne faudra jamais dépasser 20 à 25 litres d'eau pour 100 litres de crème au maximum. Plus la crème est riche, moins il faut y introduire d'eau pour un extrait sec donné du babeurre.

\section{Cas particulier de l'eau introduite au cours du barattage}

Dans tout ce qui précède, nous avons supposé l'eau introduite dans la crème une fois pour toutes et les analyses effectuées sur cette crème diluée.

Mais lorsqu'il s'agit de l'eau introduite dans la baratte, soit pour retarder la formation du grain de beurre, soit pour refroidir la crème, l'analyse ne porte pas, en général, sur la crème ainsi diluée, mais 'sur la crème avant son introduction dans la baratte, c'est-àdire sur ce que nous avons appelé la crème "initiale».

Or, il est important de connaître la quantité d'eau maximum ¿ introduire dans la baratte pour aboutir à un babeurre de richesse donnée en extrait sec - ou de calculer la quantité d'eau qui a été introduite dans la baratte en fonction de la valeur de l'extrait sec du babeurre.

Lorsqu'on baratte de la crème pure non diluée et qu'on ajoute de l'eau au cours du barattage, on produit évidemment du babeurre dilué. Le calcul de l'extrait sec de ce babeurre en fonction de la quantité d'eau introduite ou, inversement, le calcul de l'eau introduite d'après l'extrait sec du babeurre, se ramène aux condi- 
tions de l'établissement de la formule n ${ }^{0} 5$ où le calcul de l'extrait sec du babeurre a été fait en fonction des caractéristiques initiales de la crème présumée pure puisque, ici, l'analyse porte sur la crème avant son introduction dans la baratte, c'est-à-dire avant dilution.

En principe donc, connaissant la quantité d'eau introduite dans la baratte (M) exprimée en centimètres cubes par litre de crème, ainsi que les caractéristiques par litre de la crème pure initiale (A d'où $\mathrm{E}$ ) nous en déduirons l'extrait du babeurre par litre à l'aide de la formule $\mathrm{n}^{\circ} 5$ :

$$
\mathrm{e}=\frac{93 \mathrm{E}}{\mathrm{E}+0,093 \mathrm{M}}
$$

Inversement, connaissant les caractères de la crème initiale et l'extrait sec du babeurre, nous pouvons en déduire $\mathbf{M}$, c'est-à-dire la quantité d'eau introduite dans la baratte par litre de crème en appliquant la formule suivante, tirée de la précédente :

$$
\dot{M}=\frac{E(93-\mathrm{e})}{0,093 \mathrm{e}}
$$

Mais tout cela n'est correct que si la crème avant barattage est bien dans la situation d'une crème pure " initiale ", c'est-à-dire non diluée.

Or, il existe manifestement des causes de dilution de la erème, extérieures au barattage, comme nous l'avons vu.

Nous pouvons donc nous trouver - et c'est le cas le plus fréquent - dans la situation suivante :

Une crème pure a subi une première dilution $\left(\mathrm{M}_{1}\right)$ au stade de l'écrémage du fait des manipulations, des rinçages, etc.

La crème est analysée dans cet état de première dilution (matière grasse par kilogramme : $\mathrm{A}^{\prime}$; extrait dégraissé par kilogramme : $\mathrm{E}^{\prime}$ ). On peut done en déduire le taux de cette première dilution par litre ou par kilogramme, et même aussi l'extrait sec $\mathrm{e}_{1} \mathrm{du}$ babeurre qu'elle fournirait si elle ne devait plus être diluée.

Mais après l'analyse de cette crème déjà diluée, celle-ci reçoit, dans la baratte par exemple, une nouvelle dilution $\left(\mathrm{M}_{2}\right)$ définie comme la quantité d'eau ajoutée à 1 kilogramme de crème introduite dans la baratte. Les caractéristiques nouvelles de la crème ainsi diluée une seconde fois ne seront pas connues, du moins dans la pratique courante. Seul, l'extrait sec réel du babeurre final $\left(\mathrm{e}_{2}\right)$ pourra être obtenu par l'analyse.

Le problème qui se pose est alors le suivant :

Connaissant les caractères $\left(\mathrm{A}^{\prime}, \mathrm{E}^{\prime}\right)$ de la crème après la première dilution, en déduire : soit $e_{2}$ (extrait sec du babeurre final) 
connaissant, d'autre part, $\mathrm{M}_{2}$ (deuxième mouillage), soit $\mathrm{M}_{2}$ (deuxième mouillage) connaissant, d'autre part, $e_{2}$ (extrait sec du babeurre final).

Malgré sa complexité apparente, ce problème est maintenant facile à résoudre, car tous les calculs précédents ont préparé la solution.

L'extrait sec du babeurre contenu dans un kilogramme de cette crème diluée une première fois n'est autre que $\mathbf{E}^{\prime}$. Pour ramener cet extrait sec au litre de babeurre final, il suffit donc de connaître le volume de ce babeurre.

Or, au cours de l'établissement de la formule $n^{0} 7$, nous avons vu que ce volume, dans la crème diluée une première fois, était égal à :

$$
V_{1}=1.000-A^{\prime}-\frac{35}{93} \mathrm{E}^{\prime}
$$

A ce kilogramme de crème diluée une première fois, on a ajouté, au cours du barattage, une quantité d'eau égale à $\mathbf{M}_{2}$. Le volume total du babeurre final est donc égal à $V_{1}+M_{2}$ et l'extrait sec par litre du babeurre final vaut :

$$
\mathrm{e}_{2}=\frac{\mathrm{E}^{\prime}}{\mathrm{V}_{1}+\mathrm{M}_{2}} 1.000=\frac{1.000 \mathrm{E}^{\prime}}{1.000-\mathrm{A}^{\prime}-\frac{35}{93} \mathrm{E}^{\prime}+\mathrm{M}_{2}}
$$

soit, tous calculs faits :

$$
\mathrm{e}_{2}=\frac{93.000 \mathrm{E}^{\prime}}{93\left(1.000-\mathrm{A}^{\prime}\right)-35 \mathrm{E}^{\prime}+93 \mathrm{M}_{2}} \quad \text { (formule } \mathrm{n}^{\circ} \text { 11) }
$$

Cette formule donne l'extrait $e_{2} d u$ babeurre final obtenu à partir d'une crème diluée titrant $A^{\prime}$ grammes de matière grasse par kilogramme et $\mathbf{E}^{\prime}$ grammes d'extrait dégraissé par kilogramme, à laquelle on a ensuite ajouté $\mathrm{M}_{2}$ centimètres cubes d'eau par kilogrammes.

Inversement, de la formule $\mathbf{n}^{0} \mathbf{1 1}$, nous pouvons tirer $\mathbf{M}_{2}$ en fonction de $\mathrm{A}^{\prime}, \mathbf{E}^{\prime}, \mathrm{e}_{2}$.

Tous calculs faits, nous avons :

$$
\left.\mathrm{M}_{2}=\mathrm{A}^{\prime}+\mathrm{E}^{\prime}\left(0,376+\frac{1.000}{\mathrm{e}_{2}}\right)-1.000 \quad \text { (formule } \mathrm{n}^{0} 12\right)
$$

Cette formule permet de calculer la quantité d'eau introduite dans la baratte $\left(\mathbf{M}_{2}\right)$ par kilogramme de crème ayant subi ou non un premier mouillage, connaissant l'extrait sec par litre $\left(e_{2}\right) d u$ babeurre final, la matière grasse par kilogramme $\left(A^{\prime}\right)$ et l'extrait sec par kilogramme $\left(\mathbf{E}^{\prime}\right)$ de cette crème introduite dans la baratte. 


\section{Résumé et conclusions pratiques}

La dilution des crèmes par l'eau pose un eertain nombre de problèmes qu'il est facile de résoudre à l'aide des formules établies au cours de ce travail.

Les données obtenues par l'analyse et communes à tous ces problèmes sont : le taux de matière grasse par kilogramme de crème diluée $\left(\mathrm{A}^{\prime}\right)$, le taux d'extrait sec dégraissé par kilogramme de crème diluée $\left(E^{\prime}\right)$ et, éventuellement, le taux d'extrait sec dégraissé par litre de babeurre (e).

Les inconnues sont, en général : le taux de mouillage (M) de la crème initiale défini comme la quantité d'eau ajoutée à un litre ou à un kilogramme de crème pure initiale et, éventuellement, l'extrait sec dégraissé du babeurre (e).

Cela posé, on peut avoir à résoudre les divers problèmes suivants :

\section{$1^{0}$ Reconnaître qu'une crème est mouillée ou non.}

Connaissant sa richesse en matière grasse, on calcule l'extrait sec dégraissé qu'ourait cette crème si elle était pure à l'aide :

a) De la formule $\mathrm{n}^{0} \mathrm{l}: \mathrm{E}=0,09(1.000-\mathrm{A})$, qui donne l'extrait sec dégraissé par kilogramme, A étant la richesse en matière grasse par kilogramme ;

b) De la formule $n^{\circ} 2: \mathbf{E}=93-0,1016 \mathrm{~A}$, qui donne l'extrait sec dégraissé par litre, A étant la richesse en matière grasse par litre.

Si l'extrait sec dégraissé trouvé par l'analyse est inférieur d'au moins quelques grammes-à la valeur de $\mathrm{E}$ calculé ci-dessus, on peut affirmer que la crème a été mouillée. .

\section{$2^{\circ}$ Calculer le taux de mouillage d'une crème connaissant ses caractéristiques.}

Con̂naissant la richesse en matière grasse par kilogramme $\left(\mathrm{A}^{\prime}\right)$, et l'extrait dégraissé par kilogramme $\left(\mathbf{E}^{\prime}\right)$ de la crèmé diluée, on calcule, de la manière suivante, le taux de dilution de la crème initiale, c'est-à-dire la quantité d'eau (M) ajoutée :

a) A 1 kilogramme de crème pure initiale : à l'aide de la formule no 4 :

$$
M=1.000\left(\frac{90}{0,09 \mathrm{~A}^{\prime}+\mathrm{E}^{\prime}}-1\right)
$$

b) A un litre de crème pure initiale : à l'aide de la formule no 4 bis :

$$
\mathbf{M}^{\prime}=\mathbf{M} \times \mathrm{D}
$$


(D étant la densité de la crème pure initiale calculée comme il a été dit page 128.)

\section{$3^{\circ} \quad$ Calculer l'extrait sec du babeurre qui résulterait $\mathrm{du}$ barattage d'une crème diluée.}

Connaissant la richesse en matière grasse par kilogramme $\left(\mathbf{A}^{\prime}\right)$, et l'extrait dégraissé par kilogramme $\left(\mathbf{E}^{\prime}\right)$ de la crème diluée, on calcule l'extrait sec dégraissé du babeurre qu'elle peut fournir :

a) Par kilogramme de babeurre : en appliquant la formule $n^{0} 6$ :

$$
\mathrm{e}=\frac{\mathrm{E}^{\prime}}{1.000-\mathrm{A}^{\prime}} \times 1.000
$$

b) Par litre de babeurre : en appliquant la formule no 7 :

$$
\mathrm{e}=\frac{93.000 \mathrm{E}^{\prime}}{93\left(1.000-\mathrm{A}^{\prime}\right)-35 \mathrm{E}^{\prime}}
$$

$4^{\circ}$ Calculer le taux de mouillage d'une crème connaissant l'extrait dégraissé du babeurre qu'elle a fourni.

Connaissant la richesse en matière grasse par kilogramme (A') ou bien l'extrait dégraissé par kilogramme (E') T'une crème diluée ainsi que l'extrait dégraissé du babeurre par litre de babeurre (e), on calcule le taux de mouillage, c'est-à-dire la quantité d'eau. ajoutée à un kilogramme de crème initiale, en appliquant :

a) Soit la formule no 9 qui suppose la connaissance de $\mathbf{E}^{\prime}$ :

$$
M=\frac{1.000 \mathrm{E}^{\prime}(93-\mathrm{e})}{93 \mathrm{e}-\mathrm{E}^{\prime}(93-\mathrm{e})}
$$

b) Soit la formule no 10 qui suppose la connaissance de $\mathbf{A}^{\prime}$ :

$$
\mathrm{M}=\frac{90\left(1.000-\mathrm{A}^{\prime}\right)(93-\mathrm{e})}{93 \mathrm{e}+0,09 \mathrm{~A}^{\prime}(93-\mathrm{e})}
$$

On pourrait passer du taux de mouillage par kilogramme de crème initiale $(\mathrm{M})$ au taux de mouillage par litre de crème en appliquant la formule $n^{0} 4$ bis (voir plus haut).

$5^{\circ}$ Calculer le taux de mouillage à ne pas dépasser dans une crème donnée pour obtenir un babeurre d'extrait sec donné.

Les formules précédentes, qui s'appliquent à des crèmes déjà diluées dont on connaît les caractéristiques (ignorant tout de la crème pure initiale), ne peuvent être utilisées ici.

En revanche, puisque l'on part ici d'une crème pure dont on 
connaît les caractéristiqùes initiales,. on appliquera la formule $\mathrm{n}^{\circ} 5$ bis :

$$
\mathrm{M}=\frac{(1.000-\mathrm{A} / 0,915)(93-\mathrm{e})}{\mathrm{e}}
$$

où, exceptionnellement, A représente la richesse en matière grasse de la crème pure par litre et M la quantité d'eau à ajouter à un litre de crème pour obtenir un extrait sec (e) du babeurre par litre de babeurre.

On pourrait d'ailleurs utiliser le taux de matière grasse obtenu par kilogramme de crème en le transformant en taux de matière grasse par litre à l'aide de la formule suivante (voir page 129) :

$$
\mathrm{A}(\text { litre })=\frac{1.035 \mathrm{~A} \text { (kilogramme) }}{1.000+0,1311 \mathrm{~A} \text { (kilogramme) }}
$$

\section{$6^{\circ}$ Calculer la quantité d'eau introduite dans la baratte connaissant l'extrait sec du babeurre par litre ou inver- sement.}

S'il s'agissait d'une crème pure exempte d'eau introduite dans la baratte, le problème serait simple (voir p. 135 et 136), mais en général on ignore s'il s'agit d'une crème non diluée, et le plus souvent elle a déjà subi une première dilution (inconnue) au cours de l'écrémage, des rinçages, etc.

Le cas général d'une crème déjà diluée quand on l'introduit dans la baratte, sera traité de la manière suivante : connaissant sa richesse en matière grasse par kilogramme (A') et son extrait dégraissé par kilogramme $\left(\mathrm{E}^{\prime}\right)$ ainsi que la quantité d'eau $\left(\mathrm{M}_{2}\right)$ ajoutée dans la baratte par kilogramme de crème, on calculera l'extrait sec du babeurre par litre à l'aide de la formule $\mathrm{n}^{0} 11$ :

$$
\mathrm{e}_{2}=\frac{93.000 \mathrm{E}^{\prime}}{93\left(1.000-\mathrm{A}^{\prime}\right)-35 \mathrm{E}^{\prime}+93 \mathrm{M}_{2}}
$$

ou, inversement, connaissant l'extrait sec dégraissé du babeurre par litre $\left(e_{2}\right)$ on déduira la quantité d'eau ajoutée dans lá baratte par kilogramme de crème à l'aide de la formule no 12 :

$$
\mathrm{M}_{2}=\mathrm{A}^{\prime}+\mathrm{E}^{\prime}\left(0,376+\frac{1.000}{\mathrm{e}_{2}}\right)-1.000
$$

Cette formule, très générale, s'appliquerait évidemment au cas d'une crème pure introduite dans la baratte sans aucune dílution préalable.

(La partie expérimentale de ce travail a été effectuée avec la collaboration de M. DABADIE, du Laboratoire de Chimie de la Laiterie des Fermiers Réunis). 